\title{
FLAT-BAND CONDITIONS INFLUENCED BY SPIN-ORBIT INTERACTION IN THE PRESENCE OF EXTERNAL MAGNETIC FIELD
}

\author{
Nóra Kucska \\ PhD student, University of Debrecen, Department of Theoretical Physics \\ 4026 Debrecen, Bem tér 18/B, Hungary, e-mail: kucska@phys.unideb.hu

\section{Zsolt Gulácsi} \\ professor, University of Debrecen, Department of Theoretical Physics \\ 4026 Debrecen, Bem tér 18/B, Hungary, e-mail: gulacsi@phys.unideb.hu
}

\begin{abstract}
We deduct conditions for the Hamiltonian coupling strengths necessary to achieve flat bands in polymers (i.e. a pentagon chain) considering many-body spin-orbit coupling and external magnetic field. We consider itinerant electrons on pentagon chains with first neighbour hoppings, on-site electron potentials and spin-flip first neighbour hoppings representing the Rashba type spin-orbit interaction (SOI). The external magnetic field is also present in the system via the Peierls phase factors. The band structure is obtained by solving the secular equation of the diagonalized one particle part of the Hamiltonian in k-space (momentum-space). The flat band conditions make the bands k-independent, providing a highly a degenerate state, which gives broad possibilities for applications. In our work we have shown how the SOI is able to relax the strict, rigid flat band conditions given by the Hamiltonian coupling strengths. The role of the external magnetic field was also investigated.
\end{abstract}

Keywords: flat bands, conducting polymers, spin-orbit coupling

\section{Introduction}

Given by their huge degeneracy, flat bands generate a real interest since allow the appearance of several condensed phases for different systems, e.g. conducting polymers (Gulácsi et al., 2010), twisted bilayer graphene (Dong et al., 2021; Lisi et al., 2021), honeycomb optical lattice (Wu et al., 2007), nonHermitian optical lattices (Zhang et al., 2019).

In this study we first introduce the analysed system and its Hamiltonian, then transform the one particle part of the Hamiltonian to the k-space. With the transformation given, we deduct the band structure of the system by diagonalizing the one particle part of the Hamiltonian and solving the secular equation. By eliminating the k-dependent parts in the band structure, we get a highly degenerate state, called a flat band. The elimination of the k-dependent parts is obtained by the flat band conditions.

However, manufacturing flat bands in real systems seems to encounter real difficulties given by the rigidly fixed flat band conditions (strict relations between Hamiltonian parameters) they require. We show in this paper that taking many-body spin-orbit coupling (SOI) and the action of external magnetic field into account, these rigidly fixed flat band conditions can be substantially relaxed, enhancing the engineering possibilities of flat bands in real systems. 
In this paper we study conducting polymers. The base of the conducting polymer is a pentagon (e.g. polyaminotriazole type of chain). We considered two SOI strengths, first $\lambda_{c}$ on the external links and the second $\lambda$ in the base.

In order to make the model even more suitable for possible technical applications we have taken external magnetic field into account during our investigation.

\section{The system analysed}

The system analysed is a pentagon chain, with an external magnetic field perpendicular to the plane of the cell (see Fig.1,2). The unit cell of the system contains 6 sites, including the external link.

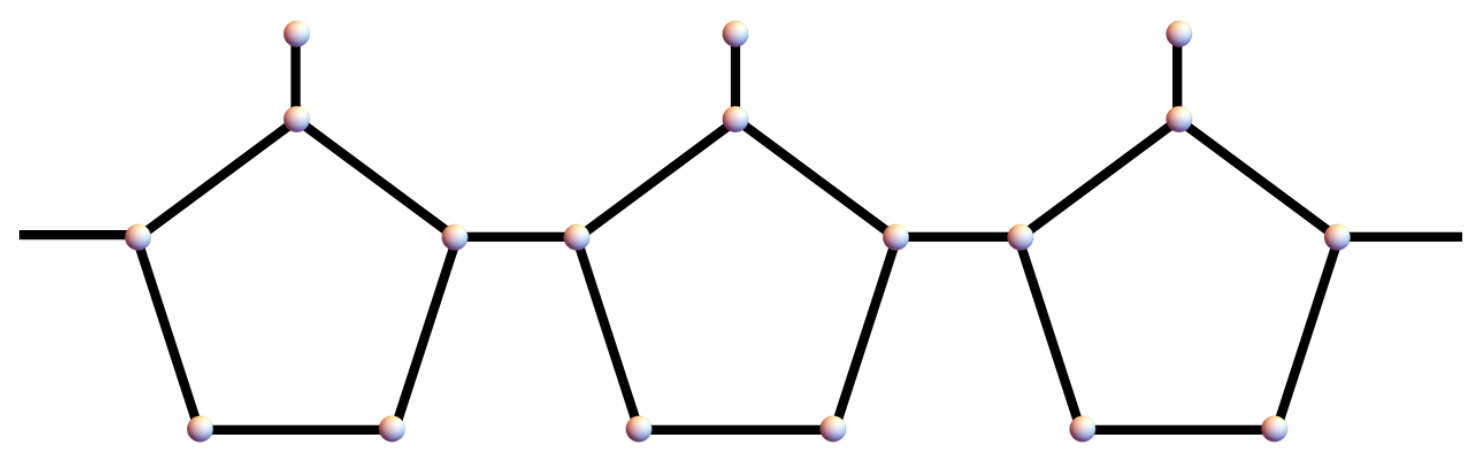

Figure 1. The pentagon chain

The Hamiltonian of the system has the form

$$
\begin{gathered}
H=\sum_{i} \sum_{\sigma, \sigma^{\prime}}\left(\bar{t}_{1,5}^{\sigma, \sigma^{\prime}} c_{i, 1, \sigma}^{\dagger} c_{i, 5, \sigma^{\prime}}+\bar{t}_{2,1}^{\sigma, \sigma^{\prime}} c_{i, 2, \sigma}^{\dagger} c_{i, 1, \sigma^{\prime}}+\bar{t}_{4,3}^{\sigma, \sigma^{\prime}} c_{i, 4, \sigma}^{\dagger} c_{i, 3, \sigma^{\prime}}+\bar{t}_{5,4}^{\sigma, \sigma^{\prime}} c_{i, 5, \sigma}^{\dagger} c_{i, 4, \sigma^{\prime}}+\right. \\
\left.\bar{t}_{c}^{\sigma, \sigma^{\prime}} c_{i+a, 7, \sigma}^{\dagger} c_{i, 4, \sigma^{\prime}}+t_{h} c_{i, 3, \sigma}^{\dagger} c_{i, 2, \sigma}+t_{f} c_{i, 6, \sigma}^{\dagger} c_{i, 5, \sigma}+\text { h.c. }\right)+\sum_{i, n, \sigma} \epsilon_{n} c_{i, n, \sigma}^{\dagger} c_{i, n, \sigma} .
\end{gathered}
$$

Where $\epsilon_{n}$ are the on-site one-particle potentials, on the site $n$. Considering the symmetry of the unit cell, we use the notations $\epsilon_{1}=\epsilon_{n=1}=\epsilon_{n=4}, \epsilon_{2}=\epsilon_{n=2}=\epsilon_{n=3}, \epsilon_{3}=\epsilon_{n=5}, \epsilon_{4}=\epsilon_{n=6}$. The neighbouring cells connect trough the Bravais vector a. In addition $n_{i, n}^{\sigma}=c_{i, \sigma}^{\dagger} c_{i, \sigma}$ is the number operator, where $c_{i, \sigma}^{\dagger}$ creates an electron on site $i$ with the spin $\sigma$. The $c_{i, \sigma}^{\dagger} c_{i, \sigma \neq \sigma}$ type hopping terms, (i.e the spinflip hopping terms) represent the Rashba type spin-orbit interaction for polymers (Li et al., 2017).

The external magnetic field $B$ is considered along the axis $\mathrm{z}$ and acts only trough the Peierls phase factor. The Peierls phase factors can be calculated via:

$$
\bar{t}_{j i}(B)=t_{j i} e^{i \frac{2 \pi}{\phi_{0}} \int_{i}^{j} \overrightarrow{A d l}}=t_{j i} e^{i \varphi_{j i}},
$$

where $\phi_{0}=\frac{h c}{e}$ is the flux quantum, $\mathrm{c}$ being the speed of light, $\mathrm{e}$ is the electron charge, $\mathrm{h}$ is the Plank constant. The $t_{j i}$ are the hopping couplings in the system without the magnetic field, $\varphi_{j i}$ are the so called Peierls factors. By calculating the Peierls phase factors, we get $\varphi_{32}=\varphi_{1}, \varphi_{21}=\varphi_{43}=\varphi_{2}, \varphi_{54}=$ $\varphi_{15}=\varphi_{3}, \varphi_{56}=\varphi_{74}=0$, where $\varphi_{l}, l=1,2,3$ has the form:

$$
\bar{t}_{32}^{\uparrow, \uparrow}=\bar{t}_{h} e^{i \varphi_{1}}, \varphi_{1}=\frac{2 \pi}{\phi_{0}}\left(-B y_{2}\right),
$$




$$
\begin{aligned}
& \bar{t}_{21}^{\uparrow, \uparrow}=t e^{i \varphi_{2}}, \bar{t}_{43}^{\uparrow, \uparrow}=t e^{i \varphi_{2}}, \bar{t}_{21}^{\uparrow, \downarrow}=\lambda e^{i \varphi_{2}}, \bar{t}_{43}^{\uparrow, \downarrow}=\lambda e^{i \varphi_{2}}, \quad \varphi_{2}=\frac{2 \pi}{\phi_{0}} B \frac{\left|y_{2}\right| b_{2}}{2}, \\
& \bar{t}_{54}^{\uparrow, \uparrow}=t e^{i \varphi_{3}}, \bar{t}_{15}=t e^{i \varphi_{3}}, \quad \bar{t}_{54}^{\uparrow, \downarrow}=-\lambda e^{i \varphi_{3}}, \bar{t}_{15}^{\uparrow, \downarrow}=-\lambda e^{i \varphi_{3}}, \quad \varphi_{3}=\frac{2 \pi}{\phi_{0}} B \frac{y_{1} b}{4} .
\end{aligned}
$$

We used the following notations for the SOI coupling strengths $\lambda=t_{5,1}^{\uparrow, \downarrow}=t_{1,5}^{\downarrow, \uparrow}=t_{1,2}^{\downarrow, \uparrow}=t_{2,1}^{\uparrow, \downarrow}=$ $t_{3,4}^{\downarrow, \uparrow}=t_{4,3}^{\uparrow, \downarrow}=t_{4,5}^{\uparrow, \downarrow}=t_{5,4}^{\downarrow, \uparrow}, t_{c}^{\uparrow, \downarrow}=\lambda_{c}$, while $t_{i, j}^{\uparrow, \downarrow}=-t_{j, i}^{\uparrow, \downarrow}$ holds. The first neighbour hopping terms are denoted by $t, t_{h}, t_{c}$ and $t_{f}$.

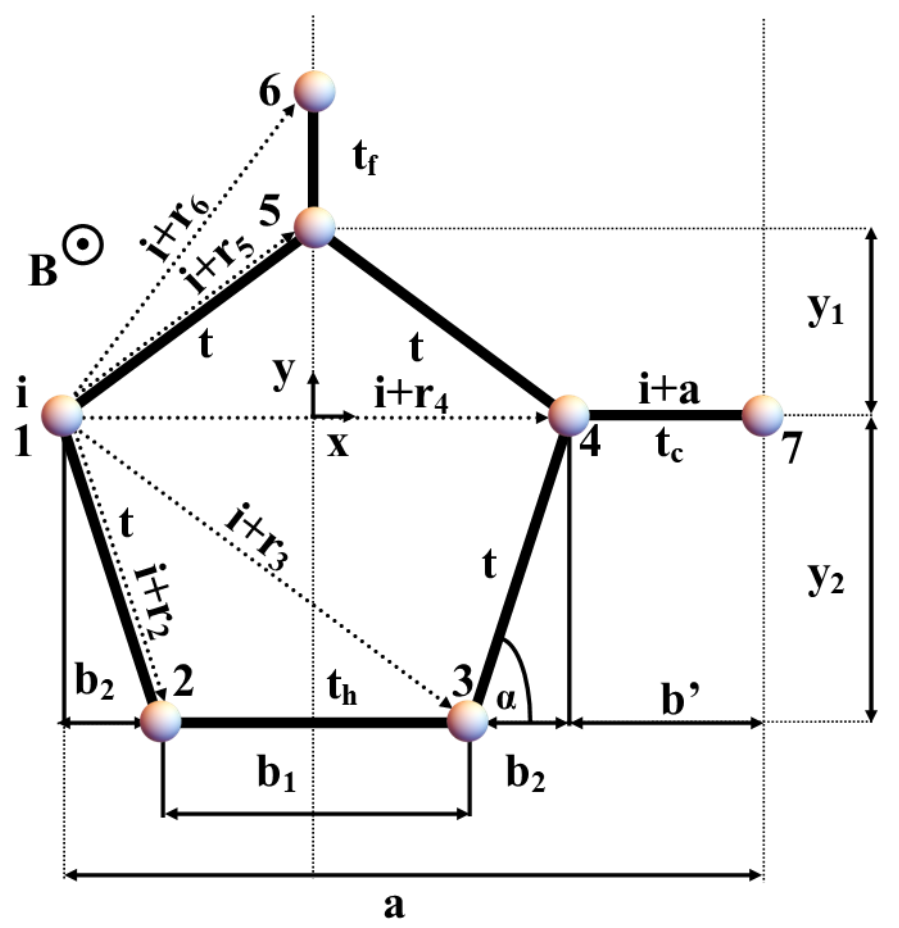

Figure 2. Unit cell defined, with in-cell notations of sites $n=1,2,3,4,5,6$.

\section{Deducing the flat band conditions}

In order to be able to study flat band conditions for the system we have to investigate the band structure, We can extract the band structure by diagonalizing the one particle part of the Hamiltonian $\left(H_{0}\right)$ in kspace and solve the secular equation.

\subsection{The Hamiltonian in k-space}

We translate $H_{0}$ to k-space, thus the fermionic operators are Fourier transformed $c_{i, r_{n}, \sigma}=$ $\frac{1}{\sqrt{N_{c}}} \sum_{k} e^{-i k\left(i+r_{n}\right)} c_{n, k, \sigma}$. Here $N_{c}$ is the number of cells and $k$ is directed along the $x$ axis (see Fig.2). The $H_{0}$ becomes: 


$$
\begin{aligned}
H_{0}=\sum_{k} \sum_{\sigma, \sigma^{\prime}} & {\left[\bar{t}_{1,5}^{\sigma, \sigma^{\prime}} c_{\boldsymbol{k}, 1, \sigma}^{\dagger} c_{\boldsymbol{k}, 5, \sigma^{\prime}} e^{i \mathbf{k}\left(\mathbf{r}_{1}-\mathbf{r}_{5}\right)}+\bar{t}_{2,1}^{\sigma, \sigma^{\prime}} c_{\boldsymbol{k}, 2, \sigma}^{\dagger} c_{i, 1, \sigma^{\prime}} e^{i \mathbf{k}\left(\mathbf{r}_{1}-\mathbf{r}_{2}\right)}+\bar{t}_{4,3}^{\sigma, \sigma^{\prime}} c_{\boldsymbol{k}, 4, \sigma}^{\dagger} c_{\boldsymbol{k}, 3, \sigma^{\prime}} e^{i \mathbf{k}\left(\mathbf{r}_{4}-\mathbf{r}_{3}\right)}\right.} \\
& +\bar{t}_{5,4}^{\sigma, \sigma^{\prime}} c_{\boldsymbol{k}, 5, \sigma}^{\dagger} c_{\boldsymbol{k}, 4, \sigma^{\prime}} e^{i \mathbf{k}\left(\mathbf{r}_{5}-\mathbf{r}_{4}\right)}+\bar{t}_{c}^{\sigma, \sigma^{\prime}} c_{\boldsymbol{k}, 1, \sigma}^{\dagger} c_{\boldsymbol{k}, 4, \sigma^{\prime}} e^{i \mathbf{k}\left(\mathbf{a}-\mathbf{r}_{4}\right)}+t_{h} c_{\boldsymbol{k}, 3, \sigma}^{\dagger} c_{\boldsymbol{k}, 2, \sigma} e^{i \mathbf{k}\left(\mathbf{r}_{3}-\mathbf{r}_{2}\right)} \\
& \left.+t_{f} c_{\boldsymbol{k}, 6, \sigma^{\prime}}^{\dagger} c_{\boldsymbol{k}, 5, \sigma} e^{i \mathbf{k}\left(\mathbf{r}_{\mathbf{6}}-\mathbf{r}_{5}\right)}+\text { h.c. }\right]+\sum_{n} \epsilon_{n} c_{\boldsymbol{k}, n}^{\dagger} c_{\boldsymbol{k}, n} .
\end{aligned}
$$

Where the exponents are (see Fig.2):

$$
\begin{gathered}
k\left(r_{4}-r_{3}\right)=k\left(r_{2}-r_{1}\right)=k b_{2}, \quad k\left(r_{6}-r_{5}\right)=0, \quad k\left(a-r_{4}\right)=k b^{\prime}, \\
k\left(r_{3}-r_{2}\right)=k b_{1}, \quad k\left(r_{5}-r_{4}\right)=k\left(r_{1}-r_{5}\right)=\frac{k b}{2} .
\end{gathered}
$$

Here we denoted the external link of the pentagon chain with $b^{\prime}$ and the length of the base with $b=$ $b_{1}+2 b_{2}$.

$$
\begin{aligned}
H_{0}=\sum_{k} \sum_{\sigma, \sigma^{\prime}}[ & \bar{t}_{1,5}^{\sigma, \sigma^{\prime}} c_{\boldsymbol{k}, 1, \sigma^{\prime}}^{\dagger} c_{\boldsymbol{k}, 5, \sigma^{\prime}} e^{i \frac{k b}{2}}+\bar{t}_{2,1}^{\sigma, \sigma^{\prime}} c_{\boldsymbol{k}, 2, \sigma}^{\dagger} c_{i, 1, \sigma^{\prime}} e^{i k b_{2}}+\bar{t}_{4,3}^{\sigma, \sigma^{\prime}} c_{\boldsymbol{k}, 4, \sigma}^{\dagger} c_{\boldsymbol{k}, 3, \sigma^{\prime}} e^{i k b_{2}} \\
& +\bar{t}_{5,4}^{\sigma, \sigma^{\prime}} c_{\boldsymbol{k}, 5, \sigma}^{\dagger} c_{\boldsymbol{k}, 4, \sigma^{\prime}} e^{i \frac{k b}{2}}+t_{h} c_{\boldsymbol{k}, 3, \sigma}^{\dagger} c_{\boldsymbol{k}, 2, \sigma} e^{i k b_{1}}+t_{f} c_{\boldsymbol{k}, 6, \sigma}^{\dagger} c_{\boldsymbol{k}, 5, \sigma}+t_{c}^{\sigma, \sigma^{\prime}} c_{\boldsymbol{k}, 1, \sigma}^{\dagger} c_{\boldsymbol{k}, 4, \sigma^{\prime}} e^{i k b^{\prime}} \\
& + \text { h.c. }]+\sum_{n} \epsilon_{n} c_{\boldsymbol{k}, n}^{\dagger} c_{\boldsymbol{k}, n} .
\end{aligned}
$$

\subsection{The band structure}

The band structure is obtained by diagonalizing the one particle part of the Hamiltonian (6)

$$
H_{0}=\sum_{k} \sum_{\sigma, \sigma}\left(c_{k, 1, \uparrow}^{\dagger}, \ldots, c_{k, 6, \uparrow}^{\dagger}, c_{k, 1, \downarrow}^{\dagger}, \ldots, c_{k, 6, \downarrow}^{\dagger}\right) \bar{M}\left(c_{k, 1, \uparrow}^{\dagger}, \ldots, c_{k, 6, \uparrow}^{\dagger}, c_{k, 1, \downarrow}^{\dagger}, \ldots, c_{k, 6, \downarrow}^{\dagger}\right)^{T},
$$

the matrix $\bar{M}$ is:

$\begin{array}{cccccccccccc}\epsilon_{1} & t e^{-i \bar{\varphi}_{2}} & 0 & t_{c} e^{i k b^{\prime}} & t e^{-i \bar{\varphi}_{3}} & 0 & 0 & -\lambda e^{-i \bar{\varphi}_{2}} & 0 & \lambda_{c} e^{i k b^{\prime}} & -\lambda e^{-i \bar{\varphi}_{3}} & 0 \\ t e^{i \bar{\varphi}_{2}} & \epsilon_{2} & t_{h} e^{-i \bar{\varphi}_{1}} & 0 & 0 & 0 & \lambda e^{i \bar{\varphi}_{2}} & 0 & 0 & 0 & 0 & 0 \\ 0 & t_{h} e^{-i \bar{\varphi}_{1}} & \epsilon_{2} & t e^{-i \bar{\varphi}_{2}} & 0 & 0 & 0 & 0 & 0 & -\lambda e^{-i \bar{\varphi}_{2}} & 0 & 0 \\ t_{c} e^{-i k b^{\prime}} & 0 & t e^{i \bar{\varphi}_{2}} & \epsilon_{1} & t e^{-i \bar{\varphi}_{2}} & 0 & -\lambda_{c} & 0 & \lambda e^{i \bar{\varphi}_{2}} & 0 & \lambda e^{i \bar{\varphi}_{3}} & 0 \\ t e^{i \bar{\varphi}_{3}} & 0 & 0 & t e^{-i \bar{\varphi}_{3}} & \epsilon_{3} & t_{f} & \lambda e^{-i \bar{\varphi}_{3}} & 0 & 0 & -\lambda e^{-i \bar{\varphi}_{3}} & 0 & 0 \\ 0 & 0 & 0 & 0 & t_{f} & \epsilon_{4} & 0 & 0 & 0 & 0 & 0 & 0 \\ 0 & \lambda e^{-i \bar{\varphi}_{2}} & 0 & -\lambda e_{c} e^{i k b^{\prime}} & \lambda e^{-i \bar{\varphi}_{3}} & 0 & \epsilon_{1} & t e^{-i \bar{\varphi}_{2}} & 0 & t_{c} e^{i k b^{\prime}} & t e^{-i \bar{\varphi}_{3}} & 0 \\ -\lambda e^{i \bar{\varphi}_{2}} & 0 & 0 & 0 & 0 & 0 & t e^{i \bar{\varphi}_{2}} & \epsilon_{2} & t_{h} e^{-i \bar{\varphi}_{1}} & 0 & 0 & 0 \\ 0 & 0 & 0 & \lambda e^{-i \bar{\varphi}_{2}} & 0 & 0 & 0 & t_{h} e^{i \bar{\varphi}_{1}} & \epsilon_{2} & t e^{-i \bar{\varphi}_{2}} & 0 & 0 \\ \lambda_{c} e^{-i k b^{\prime}} & 0 & -\lambda e^{i \bar{\varphi}_{2}} & 0 & -\lambda e^{i \bar{\varphi}_{3}} & 0 & t_{c} e^{-i k b^{\prime}} & 0 & t e^{i \bar{\varphi}_{2}} & \epsilon_{1} & t e^{i \bar{\varphi}_{3}} & 0 \\ -\lambda e^{i \bar{\varphi}_{3}} & 0 & 0 & \lambda e^{-i \bar{\varphi}_{3}} & 0 & 0 & t e^{i \bar{\varphi}_{3}} & 0 & 0 & t_{c} e^{i k b^{\prime}} & \epsilon_{3} & t_{f} \\ 0 & 0 & 0 & 0 & 0 & 0 & 0 & 0 & 0 & 0 & t_{f} & \epsilon_{4}\end{array}$




$$
\bar{\varphi}_{1}=k b_{1}+\varphi_{1}, \quad \bar{\varphi}_{2}=k b_{2}+\varphi_{2}, \quad \bar{\varphi}_{3}=\left(\frac{k b}{2}-\varphi_{3}\right) .
$$

We deduce the diagonalized energies (i.e. the bare band structure) from the secular equation of $\bar{M}$. We solve the characteristic equation, by reducing $\mathrm{B}(E,\{\mathrm{x}\},\{\operatorname{trig}(\mathbf{a k})\})=\bar{M}-I E$, where $E$ are the energy eigenvalues, $I$ is the identity matrix.

The $\{\mathrm{x}\}$ represents a set of the Hamiltonian parameters, while $\{\operatorname{trig}(\mathbf{a k})\}$ holds the trigonometric functions of the Bravais vector and contains the $\mathbf{k}$ momentum dependence of the band structure. These $\mathbf{k}$ dependent trigonometric functions in the characteristic equation are from the exponents in matrix $\bar{M}$. The band structure is given by:

$$
\mathrm{B}(E,\{\mathrm{x}\},\{\operatorname{trig}(\mathbf{a k})\})=0 .
$$

In this case the condition $\mathrm{B}(E,\{\mathrm{x}\},\{\operatorname{trig}(\mathbf{a k})\})=0$ for the matrix $\bar{M}$ can be written as:

$$
\mathrm{B}(E,\{x\}, \operatorname{trig}(\mathbf{a k}))=F_{0}+F_{1} \cos (\mathbf{a k}+\varphi)+F_{2} \sin (\mathbf{a k}+\varphi)=0,
$$

where $\varphi=\varphi_{1}+2 \varphi_{2}+\varphi_{3}$.

\subsection{The flat band conditions}

In the case of flat bands we find that the $E$ of the flat band is not dependent of the $\mathbf{k}$ momentum. The $\mathbf{k}$ dependent parts appear in the band structure in $F(\{x\}) \operatorname{trig}(\mathbf{a k})$ form(10), meaning the flat band conditions are:

$$
F(\{x\})=0 .
$$

Since $F(\{x\})$ is in function of the Hamiltonian parameters, each flat band condition will give us rigid conditions regarding the set of $\{x\}$ parameters.

During the investigation of the flat band conditions for the studied system, the origin of the energy axis is fixed to the position of the flat band, i.e. $E=0$ holds. This means that the flat band will be fixed and appear on the ox axis.

The flat band conditions for the system are:

While

$$
F_{1}=(-K v-S u), \quad F_{2}=(K u-S v) .
$$

$$
\begin{gathered}
K=\epsilon_{4} \frac{\cos \left(4 \varphi_{3}+\varphi_{b}\right)}{\epsilon_{3} \epsilon_{4}-t_{f}^{2}}-t_{h} \frac{\cos \left(2 \varphi_{3}\right)}{\epsilon_{2}^{2}-t_{h}^{2}}, \quad S=\epsilon_{4} \frac{\sin \left(4 \varphi_{3}+\varphi_{b}\right)}{\epsilon_{3} \epsilon_{4}-t_{f}^{2}}-t_{h} \frac{\sin \left(2 \varphi_{3}\right)}{\epsilon_{2}^{2}-t_{h}^{2}}, \\
v=2\left(2 \lambda \lambda_{c} t+t_{c}\left(\lambda^{2}-t^{2}\right)\right), \quad u=2\left(-2 \lambda t t_{c}+\lambda_{c}\left(\lambda^{2}-t^{2}\right)\right),
\end{gathered}
$$

where $\varphi_{b}=\varphi_{1}+2 \varphi_{2}$.

The solution for $F_{1}=0, F_{2}=0$ can be summarized as $K=0, S=0$, since other seemingly possible solutions would lead us to complex $\lambda, \lambda_{c}$ coupling strengths which are not physical solutions for our $1 \mathrm{D}$ system with Rashba spin-orbit coupling. 
The $K=0, S=0$ gives restrictions for the Peierls phase factors, thus restriction for the external magnetic field values trough (3):

or

$$
\begin{aligned}
& \varphi_{\left.b\right|_{1}}=2 \arctan \left(\cot \left(\varphi_{3}\right)\right)+2 \pi m, \quad \varphi_{\left.b\right|_{2}}=-2 \arctan \left(\tan \left(\varphi_{3}\right)\right)+2 \pi m, \\
& \text { while } \tan \left(\varphi_{\left.3\right|_{1,2}}\right)\left(\tan ^{2}\left(\varphi_{\left.3\right|_{1,2}}\right)-1\right) \neq 0, \quad \frac{2 \varphi_{\left.3\right|_{1,2}}-\pi}{2 \pi} \notin Z \text {, } \\
& \left(\varphi_{\left.b\right|_{3}}=2 \pi n, \quad \varphi_{\left.3\right|_{3}}=\frac{1}{2}(\pi+2 \pi m)\right), \\
& \left(\varphi_{\left.b\right|_{4}}=\pi+2 \pi n, \quad \varphi_{\left.3\right|_{4}}=\pi m\right), \\
& \left(\varphi_{\left.b\right|_{5}}=\pi+2 \pi n, \quad \varphi_{\left.3\right|_{5}}=\frac{1}{2}(\pi+2 \pi m)\right) \text {, }
\end{aligned}
$$

where $\mathrm{m}, \mathrm{n} \in \mathrm{Z}$.

While for each solution $\epsilon_{4} \frac{1}{\epsilon_{3} \epsilon_{4}-t_{f}^{2}}=i_{\varphi} t_{h} \frac{1}{\epsilon_{2}^{2}-t_{h}^{2}}$ stands. These Peierls phase factors can be categorized into two groups, by the value of $i_{\varphi}$; for the solutions $\left(\varphi_{\left.b\right|_{2}}, \varphi_{\left.3\right|_{2}}\right),\left(\varphi_{\left.b\right|_{5}}, \varphi_{\left.3\right|_{5}}\right), i_{\varphi}=1$, while for the $\left(\varphi_{\left.b\right|_{1}}, \varphi_{\left.3\right|_{1}}\right),\left(\varphi_{\left.b\right|_{3}}, \varphi_{\left.3\right|_{3}}\right),\left(\varphi_{\left.b\right|_{4}}, \varphi_{\left.3\right|_{4}}\right) i_{\varphi}=-1$.

The $i_{\varphi}=1$ solutions are consistent with the solutions without external magnetic fields (i.e a system with both $\varphi_{3}$ and $\varphi_{b}$ values zero). In this case the flat band condition is:

$$
\epsilon_{4} \frac{1}{\epsilon_{3} \epsilon_{4}-t_{f}^{2}}=t_{h} \frac{1}{\epsilon_{2}^{2}-t_{h}^{2}} .
$$

This gives us a restriction for the value of $t_{f}$ :

$$
t_{\left.f\right|_{1,2}}= \pm \frac{\sqrt{-\epsilon_{4}\left(\epsilon_{2}^{2}-t_{h}^{2}\right)+\epsilon_{3} \epsilon_{4} t_{h}}}{\sqrt{t_{h}}}
$$

For the solutions with $i_{\varphi}=-1$, the flat band condition is:

$$
\epsilon_{4} \frac{1}{\epsilon_{3} \epsilon_{4}-t_{f}^{2}}=-t_{h} \frac{1}{\epsilon_{2}^{2}-t_{h}^{2}} .
$$

And the restriction for $t_{f}$ becomes:

$$
t_{\left.f\right|_{1,2}}= \pm \frac{\sqrt{\epsilon_{4}\left(\epsilon_{2}^{2}-t_{h}^{2}\right)+\epsilon_{3} \epsilon_{4} t_{h}}}{\sqrt{t_{h}}} .
$$


Besides the flat band conditions, there is another restriction to satisfy (10), for that part of the secular equation which does not contain $\mathbf{k}$ dependence. We denoted this condition with $F_{0}$ :

$$
\begin{gathered}
F_{0}=A^{2}-\lambda_{c}{ }^{2}-t_{c}{ }^{2}-\left(\lambda^{2}+t^{2}\right)^{2}\left(\frac{\epsilon_{4}{ }^{2}}{\left(\epsilon_{3} \epsilon_{4}-t_{f}{ }^{2}\right)^{2}}+\frac{t_{h}{ }^{2}}{\left(\epsilon_{2}{ }^{2}-t_{h}\right)^{2}}-2 \cos \left(2 \varphi_{3}+\varphi_{b}\right) \frac{\epsilon_{4} t_{h}}{\left(\epsilon_{2}{ }^{2}-t_{h}{ }^{2}\right)\left(\epsilon_{3} \epsilon_{4}-t_{f}{ }^{2}\right)}\right), \\
A=\epsilon_{1}-\left(t^{2}+\lambda^{2}\right)\left(\frac{\epsilon_{4}}{\epsilon_{3} \epsilon_{4}-t_{f}{ }^{2}}+\frac{\epsilon_{2}}{\epsilon_{2}{ }^{2}-t_{h}{ }^{2}}\right) .
\end{gathered}
$$

Dividing the solution of $F_{0}=0$ by the value of $i_{\varphi}$, we notice that for those $\left(\varphi_{b}, \varphi_{3}\right)$ solutions which belong in the group $i_{\varphi}=1$ the $F_{0}=0$ equation is once again consistent with the system without external magnetic field. In this case the value of $2 \cos \left(2 \varphi_{3}+\varphi_{b}\right)=1$, and one can easily use this condition to calculate the $\lambda_{c}$ or $\lambda$ value.

The solution of $F_{0}=0$, while $i_{\varphi}=-1$ differ from the previous. In this case the value of $2 \cos \left(2 \varphi_{3}+\varphi_{b}\right)=-1$, and we get different two $\lambda_{c}$ or $\lambda$ values, as it has been shown on Fig. 3 .

To see, how the magnetic field, and the SOI can change the rigid flat-band conditions first we turned our attention to investigate the flat band conditions for the system without SOI and external magnetic field.

In this case from the flat band conditions (12) $F_{2}$ is automatically zero, since $\lambda_{c}, \lambda, \varphi_{3}, \varphi_{b}$ are zero, while for $F_{1}$ we have:

$$
\begin{gathered}
F_{1}=-K v . \\
K=\epsilon_{4} \frac{1}{\epsilon_{3} \epsilon_{4}-t_{f}^{2}}-t_{h} \frac{1}{\epsilon_{2}^{2}-t_{h}^{2}}, \quad v=-2 t^{2} t_{c}
\end{gathered}
$$

Since the first neighbour hopping coupling strengths $t, t_{c}$ are nonzero numbers, the solution for the condition $F_{1}=0$ is $K=0$, which in agreement with the solution seen in (15). I.e the solution for the system with SOI without external magnetic field is the same at $i_{\varphi}=1$. This means that for the system without magnetic field or SOI the flat band condition for $t_{f}$ remains (16). For the condition $F_{0}=0$ we get:

$$
\begin{gathered}
F_{0}=A^{2}-t_{c}{ }^{2}-t^{4}\left(\epsilon_{4} \frac{1}{\epsilon_{3} \epsilon_{4}-t_{f}^{2}}-t_{h} \frac{1}{\epsilon_{2}^{2}-t_{h}^{2}}\right)^{2}, \\
A=\epsilon_{1}-t^{2}\left(\frac{\epsilon_{4}}{\epsilon_{3} \epsilon_{4}-t_{f}{ }^{2}}+\frac{\epsilon_{2}}{\epsilon_{2}^{2}-t_{h}^{2}}\right) .
\end{gathered}
$$

This was used to calculate the value of $t_{c}$ :

$$
t_{\left.c\right|_{1,2}}= \pm \frac{\left(\epsilon_{2}+t_{h}\right)\left(\epsilon_{1}\left(\epsilon_{2}-t_{h}\right)-t^{2}\right)}{\sqrt{\left(\epsilon_{2}^{2}-t_{h}^{2}\right)^{2}}}
$$

These solutions for the flat band conditions for the system without SOI and magnetic field are known and are in agreement with the literature (Gulácsi, 2013). The latter strict condition for the value of $t_{c}$ can be evaded, by changing the SOI coupling of the system. If we change the strict, rigid values for $t_{c}$ given by the flat band conditions for the system without SOI and external magnetic field, Eq.(22) by $\Delta t_{c}$, the flat band becomes a dispersive band. The flat band can be achieved again by changing the $\lambda_{c}$ 
or $\lambda$ value of the system to a value which can be calculated from (19). (see Fig.3)
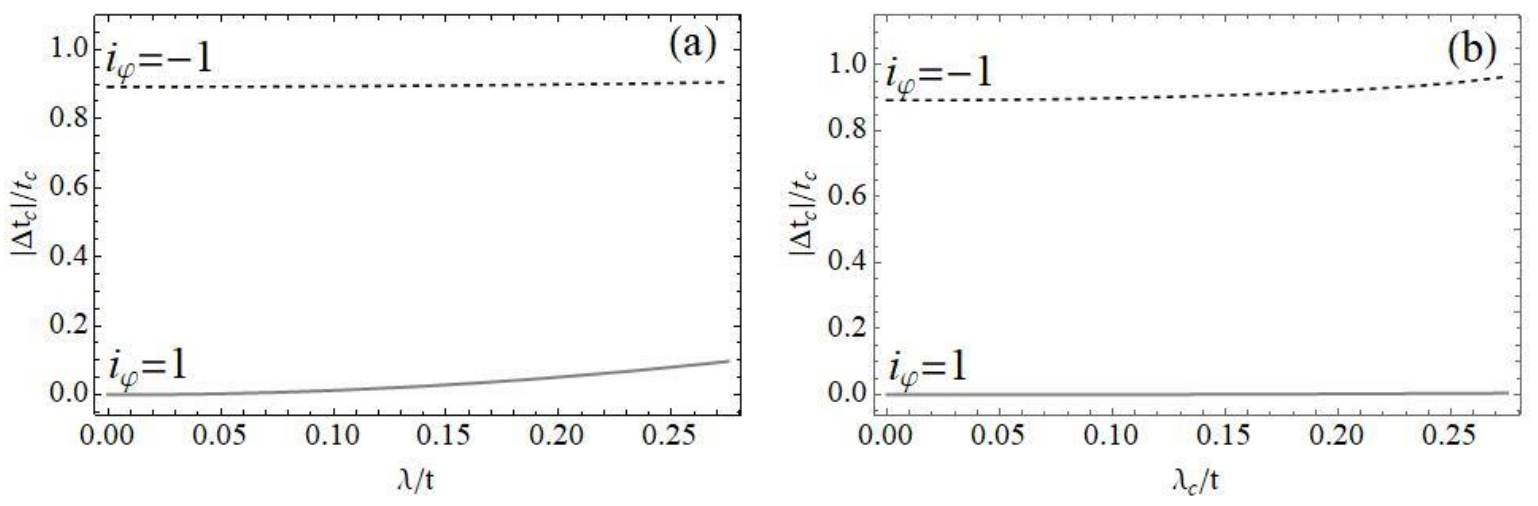

Figure 3. The strict, rigid values for $t_{c}$ given by the flat band conditions for the system without SOI and external magnetic field (22) are changed by $\Delta t_{c}$. The flat band achieved by a) $\left.\lambda / t b\right) \lambda_{c} / t$ provided by the flat band conditions for the system with SOI and magnetic field (19). The $i_{\varphi}=1$ curve is congruent with the system with SOI and without magnetic field. The H parameters are $\epsilon_{1}=$ $0.77, \epsilon_{2}=1.19, \epsilon_{3}=0.72, \epsilon_{4}=0.26, t_{h}=0.9, t_{c}=2.7$ and are given in the first neighbour hopping $t$ units. The $t_{f}=0.12$ for $i_{\varphi}=1$, is calculated from (15), $t_{f}=0.6$ for $i_{\varphi}=-1$ is calculated from (18).

\section{Summary}

We worked out the band structure for an itinerant electron system in polymer chains, with first neighbour hopping terms, first neighbour spin-flip hopping terms (which are present in the system due to Rashba type spin-orbit interaction), on-site electron potential and external magnetic field. We calculated the strict restrictions between Hamiltonian coupling strengths necessary to obtain flat bands in the case of no magnetic field or SOI.

Exemplifying our obtained results in the case of conducting polymers, we show that taking the action of the many-body spin-orbit interaction in the presence of external magnetic fields into account, the rigidly fixed flat band conditions can be substantially relaxed, allowing an easier creation in practice of flat bands in real systems. This result seems to be advantageous from the point of view of technological applications, since the SOI coupling strengths can be continuously tuned by external electric field. We also found that flat bands can also appear in the presence of external magnetic field, which further expands possibilities of technical applications.

\section{Acknowledgement}

N. K. acknowledges the support of ÚNKP-21-3-II New National Excellence Program of the Hungarian Ministry of Human Capacities.

\section{References}

[1] Gulacsi, Z., Kampf, A., Vollhardt, D. (2010). Route to ferromagnetism in organic polymers. Phys. Rev. Lett. 105, 266403. https://doi.org/10.1103/PhysRevLett.105.266403 
[2] Dong, K., Zhang, T., Li, J., Wang, Q., Yang, F., Rho, Y., Wang, D., Grigoropoulos, C. P., Wu, J., Yao, J. (2021). Flat bands in magic-angle bilayer photonic crystals at small twists. Phys. Rev. Lett. 126, 223601. https://doi.org/223601.10.1103/PhysRevLett.126.223601

[3] Lisi, S. et al. (2021). Observation of flat bands in twisted bilayer graphene. Nature Physics, 17, 189-193. https://doi.org/10.1038/s41567-020-01041-x

[4] Wu, C., Bergman, D., Balents, L., Das Sarma, S. (2007). Flat bands and wigner crystallization in the honeycomb optical lattice. Phys. Rev. Lett. 99, 070401, 1. https://doi.org/10.1103/PhysRevLett.99.070401

[5] Zhang, S. M., Jin, L. (2019). Flat band in two-dimensional non-Hermitian optical lattices. Phys. Rev. A 100, 043808. https://doi.org/10.1103/PhysRevA.100.043808

[6] Li, H. et al. (2017). Research of spin-orbit interaction in organic conjugated polymers, IOP Conf. $\begin{array}{lllll}\text { Series: } \quad \text { Materials } & \text { Science and Engineering } 213 & 012005 .\end{array}$ https://doi.org/10.1088/1757-899X/213/1/012005

[7] Gulácsi, Z. (2013). Exact Ground States of Correlated Electrons on Pentagon Chains. International Journal of Modern Physics B, 27(14) 1330009, https://doi.org/10.1142/S0217979213300090 